\title{
Sistema De Monitoreo de Variables Medioambientales Usando Una Red de Sensores Inalámbricos y Plataformas De Internet De Las Cosas
}

\section{(Monitoring System of Environmental Variables Using a Wireless Sensor Network and Platforms of Internet of Things)}

\author{
Manuel Quiñones-Cuenca ${ }^{1,2}$, Víctor González-Jaramillo', Rommel Torres ${ }^{1}$, Miguel Jumbo
}

\begin{abstract}
Resumen:
En este trabajo se propone un sistema para la recolección de datos meteorológicos usando una Red de Sensores Inalámbricos (RSI), capaz de transmitir los datos en tiempo real. El sistema logra automatizar los procesos de obtención de datos de manera continua y a largo plazo, por medio de un módulo de abastecimiento de energía solar que permite autonomía para su funcionamiento. Para la viabilidad del diseño e implementación de prototipos se propone la construcción de dos sistemas basados en DigiMesh y Wi-Fi, los que se pueden aplicar a diferentes escenarios como zonas urbanas y rurales. Adicionalmente se evalúa la transmisión de información a plataformas de Internet de las Cosas (IoT), en donde se gestionará y visualizará los datos obtenidos por los nodos. Este sistema fue concebido como una alternativa de bajo costo comparado con estaciones meteorológicas convencionales que posean estas prestaciones y está basado en componentes de hardware y software libre. Finalmente, se realizó la validación de los resultados obtenidos mediante un análisis estadístico con los datos registrados en una estación meteorológica Davis Vantage Pro, obteniendo un error relativo promedio máximo de $4,93 \%$.
\end{abstract}

Palabras clave: Estación meteorológica; medioambiente; RSI; loT; Wi-Fi.

\begin{abstract}
:
This work proposes a system for collecting meteorological data using a Wireless Sensor Network (WSN), that is able to transmit data in real-time. The system automatizes the process of collecting the data in a continuous manner for long periods of time, for this, the module is equipped with a source of solar energy that allows autonomous operation. In order to obtain viability of design and prototype implementation, the construction of two systems was proposed based on DigiMesh and Wi-Fi; those prototypes could be applied to different scenarios such as urban and rural areas. Additionally, it was performed an evaluation of broadcasting of information to platforms of Internet of Things (IOT), where the data collected by the nodes will be managed and displayed. This system was conceived as a low-cost alternative compared with conventional weather stations that offer these facilities and are based on free hardware and software components. Finally, the validation of the obtained results was performed using a statistical analysis with the collected data of the weather station Davis Vantage Pro, obtaining a maximum average relative error of $4.93 \%$.
\end{abstract}

Keywords: Environment; loT; weather stations; Wi-Fi; WSN.

\footnotetext{
${ }^{1}$ Universidad Técnica Particular de Loja, Loja - Ecuador (\{mfquinonez, vhgonzalez, rovitor, majumbo2\} @utpl.edu.ec)

2 Universidad Nacional de Loja, Loja - Ecuador (mfquinonez@unl.edu.ec)
} 


\section{Introducción}

Hoy en día con las Tecnologías de la Información y Comunicación (TIC), se ha logrado adelantos importantes en la recolección, análisis y distribución de información relacionada con el estado climático, monitoreo de variables medioambientales, estudios de biodiversidad, entre otras. Esta información tiene distintos fines, entre ellos, la preservación digital de datos, su representación gráfica y el modelamiento de patrones climatológicos. Algunas iniciativas se enfocan en mejorar la calidad de vida de las personas, mediante la obtención y estimación de factores que afectan el entorno del ser humano, por ejemplo, infraestructura y transporte, agricultura, datos de la biodiversidad del sitio observado, entre otros.

Conceptos como Ciudades Inteligentes o Territorios Inteligentes (Smart Cities y Smart Lands), se apoyan en las TICs, especialmente en las plataformas de Internet de las Cosas (IOT) por medio de las cuales se puede obtener datos de variables medioambientales y poder proyectar y mejorar aspectos propios de las ciudades o territorios.

Uno de los retos que deben soportar las Ciudades Inteligentes está altamente relacionado con la infraestructura de comunicación necesaria para la recolección y distribución de información. En estos espacios se emplean redes de sensores inalámbricos (RSI) las cuales por su naturaleza inalámbrica y autónoma pueden ser instaladas con rapidez, además para su implementación no es necesaria una mayor inversión en adecuación de orden físico y se tiene un bajo impacto de afectación al medioambiente (Faludi, 2011, 11; Lin et al., 2011).

En este trabajo se plantea el diseño, integración e implementación de un sistema para la recolección de datos meteorológicos, para ello se usa una RSI basada en DigiMesh, lo cual permite tener una mayor flexibilidad; también se usa Wi-Fi para expandir la red de comunicaciones. El sistema emplea herramientas de hardware y software abiertos con el fin de reducir costos y se integra con plataformas de loT para la visualización y gestión de los datos. La importancia del desarrollo del sistema radica en que se minimiza los costos de implementación, operación y gestión, en comparación con estaciones meteorológicas comerciales existentes. El sistema además ofrece un modelo escalable y funcional debido a que se puede agregar nuevos módulos para la adquisición de variables adicionales, y utiliza recursos naturales como fuente de energía.

La parte restante de este documento está estructurado de la siguiente manera: en la sección 2 de Metodología se plantea la arquitectura del sistema, hardware, el abastecimiento de energía y las plataformas de IoT utilizadas. En la sección 3 de Resultados y Discusión se muestra la implantación del prototipo de una RSI con la transmisión de datos a plataformas loT y los resultados obtenidos mediante un análisis de los datos. Finalmente se presentan las Conclusiones y Recomendaciones. 


\section{Metodología}

Para desarrollar un prototipo del sistema se ha seguido una metodología de avance por fases.

La primera fase, implantación, comprende la recolección de información, específicamente, la identificación de variables que el prototipo requiere como mínimo para la recolección de datos meteorológicos, con el fin de obtener las características necesarias que permitan el dimensionamiento tanto de los sensores como de los módulos de comunicación.

La segunda fase, recolección de datos, en donde se realiza los algoritmos y la caracterización de la tarjeta de adquisición. En esta fase también se realiza la integración de los sensores con la placa base de adquisición y el análisis del consumo energético del prototipo.

Finalmente, en la tercera fase, distribución y presentación, está enfocada a la transmisión y recepción de información del prototipo, como también a la visualización y la validación de los datos en plataformas loT.

\subsection{Arquitectura del sistema}

Para la arquitectura del sistema se plantea usar dos variantes de RSI:

- La primera basada en DigiMesh para la comunicación. Está red se compone de nodos sensores y un nodo central que está conectado a una puerta de enlace (ver Figura 1.a), este usa Wi-Fi para el reenvió de datos a plataformas de loT (ver Figura 1.c).

- La segunda donde los nodos usan Wi-Fi para la comunicación. Estos integran las funcionalidades de adquisición de variables meteorológicas, procesamiento y la transmisión de datos directamente a las plataformas de loT (ver Figura 1.b). En esta variante se suprime el uso de los nodos centrales.

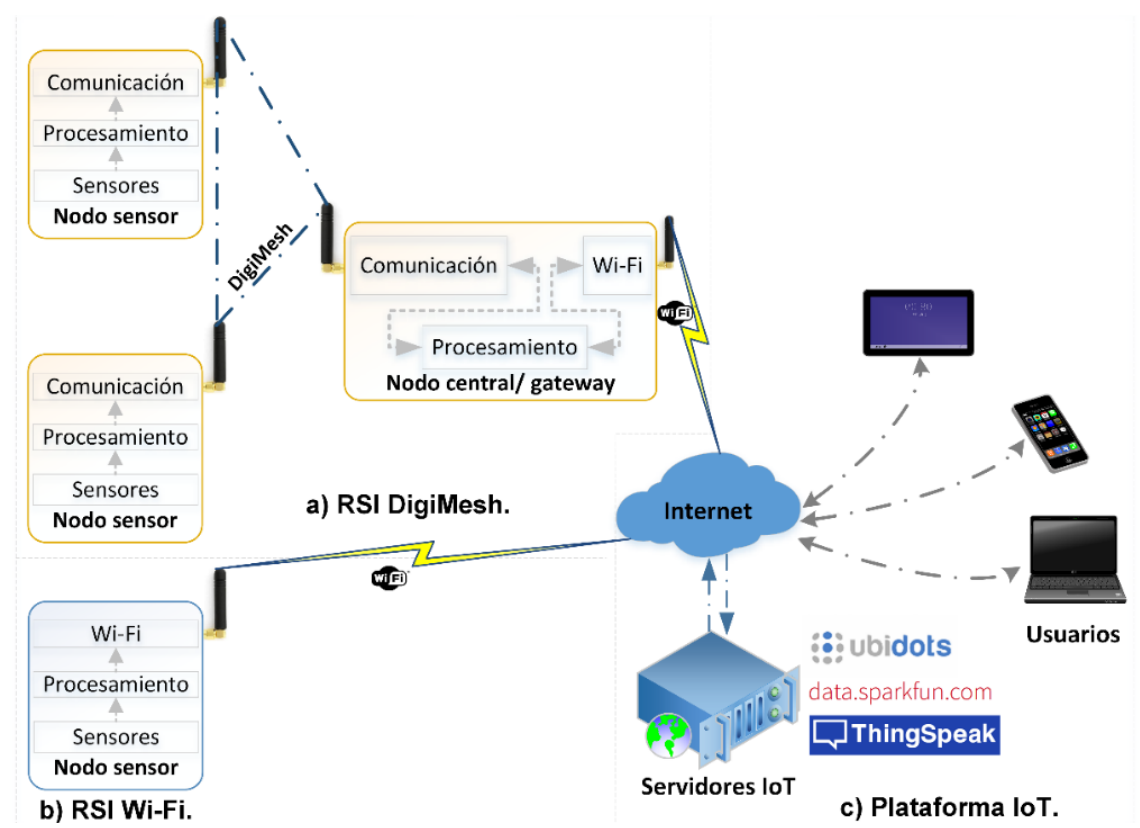

Figura 1. Arquitectura del sistema. 


\subsubsection{Nodo Sensor}

Los nodos sensores están formados por 3 módulos: sensores, procesamiento y comunicación (ver Figura 2). Estos nodos tienen por función recolectar los valores adquiridos por los sensores, procesar y enviar los datos ya sea usando Wi-Fi o DigiMesh según el modelo de RSI seleccionado.

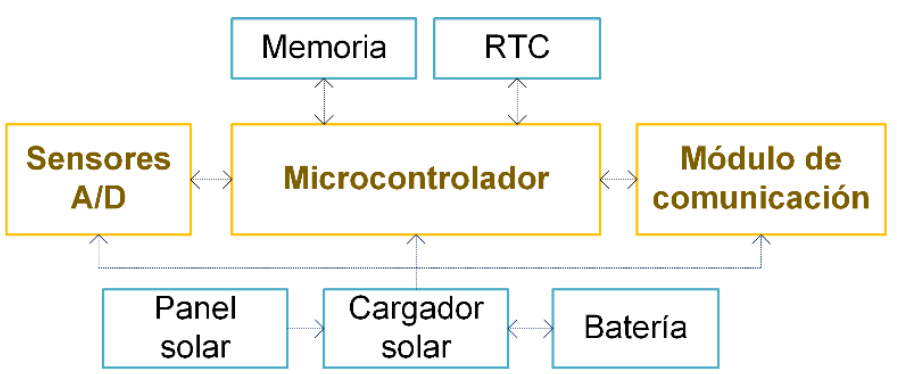

Figura 2. Arquitectura hardware del nodo sensor.

En la RSI usando DigiMesh, existe además un nodo central conformado por dos módulos de comunicación, el primero interactúa con los nodos sensores para la entrega de los valores recolectados, y el segundo retransmite la información obtenida de los nodos sensores a una plataforma IoT (Perera, Liu \& Jayawardena, 2015).

\subsection{Hardware}

Los componentes de hardware usados para la implementación de los nodos prototipo se detallan en las siguientes secciones.

\subsubsection{Sensores}

Para determinar los sensores a emplear en la adquisición de las variables meteorológicas se considera los rangos de medición y protocolos de comunicación como se indica en Tabla 1. Las variables a monitorear son: velocidad del viento, dirección del viento, lluvia instantánea, presión barométrica, temperatura ambiental, humedad relativa ambiental, temperatura del suelo, y humedad relativa del suelo. Estas son determinadas por la Organización Mundial de Meteorología (OMM) (OMM, 2014)

Tabla 1. Especificaciones de los sensores.

\begin{tabular}{|c|c|c|c|c|}
\hline Sensor & Dispositivo & Protocolo Comunic. & Rango de Medición & Exactitud \\
\hline Anemómetro & \multirow{3}{*}{ SEN-08942 } & \multirow{4}{*}{ 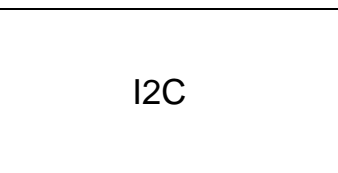 } & $2,4-228 \mathrm{~km} / \mathrm{h}$ & \\
\hline Veleta & & & $0-360^{\circ}$ & \\
\hline Pluviómetro & & & $0,2794 \mathrm{~mm}$ & \\
\hline Barómetro & MPL3115A2 & & $20-110 \mathrm{kPa}$ & $\overline{\mp 0,4 \mathrm{kPa}}$ \\
\hline Temperatura ambiente & \multirow{2}{*}{ RHT03 } & \multirow[b]{2}{*}{ 1Wire } & -40 a $80^{\circ} \mathrm{C}$ & $\mp 0,5$ \\
\hline $\begin{array}{l}\text { Humedad relativa } \\
\text { ambiente }\end{array}$ & & & $0-100 \% \mathrm{HR}$ & $\mp 2 \% \mathrm{HR}$ \\
\hline Temperatura suelo & \multirow[t]{2}{*}{ SHT10 } & \multirow{2}{*}{ 2Wire } & -40 a $123^{\circ} \mathrm{C}$ & $\mp 0,5$ \\
\hline Humedad relativa suelo & & & $0-100 \% \mathrm{HR}$ & $\mp 4,5 \% \mathrm{HR}$ \\
\hline
\end{tabular}


Adicionalmente, se requiere del módulo Weather Shield (DEV-12081), que permite el acondicionamiento de las señales de los sensores (anemómetro, veleta, pluviómetro) e integra un barómetro, un sensor de temperatura y humedad, que sirven para el monitoreo del estado de los nodos sensores.

\subsubsection{Procesamiento}

El procesamiento del prototipo se realiza en una tarjeta Arduino Uno (RedBoard DEV-12757), que es la encargada de adquirir y procesar toda la información obtenida por los sensores y administrar parte de la comunicación.

\subsubsection{Módulos de comunicación}

El sistema se diseña para ambientes rurales o urbanos con bandas ISM de $900 \mathrm{MHz}$ y $2,4 \mathrm{GHz}$. Se propone usar Wi-Fi para la transmisión de datos a Internet en ambientes urbanos donde existe una alta densidad de despliegue de esta tecnología.

Para la comunicación entre el nodo sensor y central como se indica en la figura 1a se usa módulos con protocolos de comunicación DigiMesh que permiten establecer topologías punto a punto, punto a multipunto y malla; hasta una distancia de $40 \mathrm{~km}$ empleando antenas de alta ganancia en la banda de $900 \mathrm{MHz}$. A continuación, se detalla cada uno de los elementos usados para la comunicación:

- Wi-Fi: Electric Imp. 01 (Nodo usando Wi-Fi y el nodo central) en la banda 2,4 GHz.

- Electric Imp Shield (DEV-12887), permite integrar el módulo de comunicación con la plataforma Arduino.

- DigiMesh: XBee PRO 900HP (Potencia máxima: 250mW).

- Antena dipolo de $3 \mathrm{dBi}$ para evaluar en ambiente de laboratorio. Para desplegar el sistema en un ambiente externo se usa una antena Yagi de 14dBi para el nodo sensor y para el coordinador una antena omnidireccional de $8 \mathrm{dBi}$.

\subsubsection{Abastecimiento de energía}

Para el abastecimiento de energía de los nodos se usa paneles solares, baterías LiPo y cargador solar. A continuación, se detalla los componentes usados:

- Panel solar: PRT-09241.

- Batería: LiPo 3.7V 6600mAh (ID: 353)

- Cargador solar para baterías LiPo: PRT-12885. 


\subsection{Plataformas loT}

Actualmente, han surgido una diversidad de plataformas en respuesta al rápido incremento de dispositivos inalámbricos aplicados a la medición y automatización de procesos industriales y residenciales. Las plataformas loT permiten el registro de datos adquiridos por sensores, el enrutamiento de datos en tiempo real y el control remoto de dispositivos. Además, proveen una interfaz como punto de acceso a sus recursos a través de protocolos de Internet; que permiten interactuar con lenguajes de alto nivel como LabView, Matlab y Python, para realizar procesamiento de datos y accionar remotamente un dispositivo (Wong \& Kerkez, 2016).

Debido a que usan distintas estrategias y protocolos de aplicación como Hipertext Transfer Protocol (HTTP) y Message Queue Telemetry Transport (MQTT), el prototipo se ha evaluado en tres plataformas loT: Ubidots (UBIDOTS, 2014), Phant (PHANT, 2015) y ThingSpeak (c, 2016).

\subsubsection{Ubidots}

En la figura 3a se muestra la arquitectura de la plataforma Ubidots, la que integra los flujos de datos en un entorno de computación en la nube, para crear aplicaciones que capturan información del mundo real. Ubidots provee una clave API a cada usuario que se utiliza como identificador de autenticación cuando se transmite los datos de los sensores a la nube (UBIDOTS, 2014). Esta permite registrar hasta 30000 datos de forma gratuita por mes, si se requiere registrar más datos al mes, se puede optar por pagar un plan de servicio que ofrece está plataforma según sea necesario (Bolivar \& Alexandre Da Silva, 2015).

Las principales características de la plataforma Ubidots son:

- Capacidad para publicar los datos del dispositivo sobre una API REST.

- Flexibilidad, ya que permite a sus usuarios mezclar diferentes flujos de datos y presentarlos en esta plataforma.

- Disponibilidad ubicua, debido a que está basado en la nube.

- Seguridad basada en mecanismos incorporados en la plataforma de Ubidots (autenticación mediante API o tokens).

\subsubsection{Phant}

Es una herramienta de código abierto de registro modular desarrollada por SparkFun Electronics para la recolección de datos de loT. Para el almacenamiento se requiere realizar la creación de un flujo de datos, que proporciona una clave pública y una privada. Estas claves se usan en la publicación de los datos por lo que es necesario añadirla a la trama. Para registrar los datos al servidor se puede usar la biblioteca Phant o mediante un requerimiento HTTP utilizando cualquier cliente web. Aunque es gratuito tiene un límite de 100 peticiones cada 15 minutos y tiene una 
capacidad máxima de 50MB después de sobrepasar este límite, los datos más antiguos se eliminan. Estas limitaciones se pueden eliminar si se instala en un servidor privado. En la figura $3 \mathrm{~b}$ se representa el funcionamiento de la plataforma Phant (PHANT, 2015).

\subsubsection{ThingSpeak}

Es un servicio de plataforma de análisis de loT que permite almacenar y recuperar los datos de sensores en la nube y desarrollar aplicaciones de loT. El servicio ThingSpeak también permite realizar análisis en línea y actuar sobre sus datos. Los datos del sensor pueden ser enviados a ThingSpeak desde cualquier hardware que pueda comunicarse usando una API REST o usando el protocolo MQTT. MQTT es extremadamente simple y ligero, para la publicación y suscripción; diseñado para dispositivos que están limitados por el bajo ancho de banda y alta latencia (Asghar \& Mohammadzadeh, 2015). En la Figura $3 c$ se representa la arquitectura de esta plataforma (MATHWORKS, 2016).

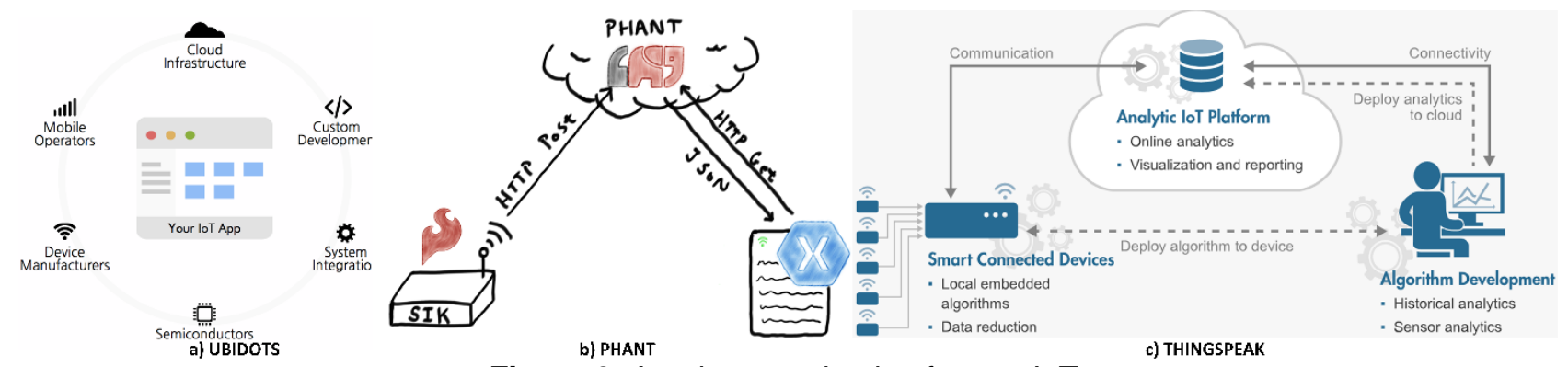

Figura 3. Arquitectura de plataformas loT.

\subsection{Dimensionamiento de autonomía de energía}

Para determinar el consumo de energía del prototipo (ver Figura 4) se realiza las mediciones en cada uno de los nodos de la RSI DigiMesh y de la RSI Wi-Fi (ver Tabla 2), y el requerimiento diario de potencia. Estas mediciones permiten establecer la capacidad de la batería (considerando una autonomía de 2 días), y el dimensionamiento del panel solar.

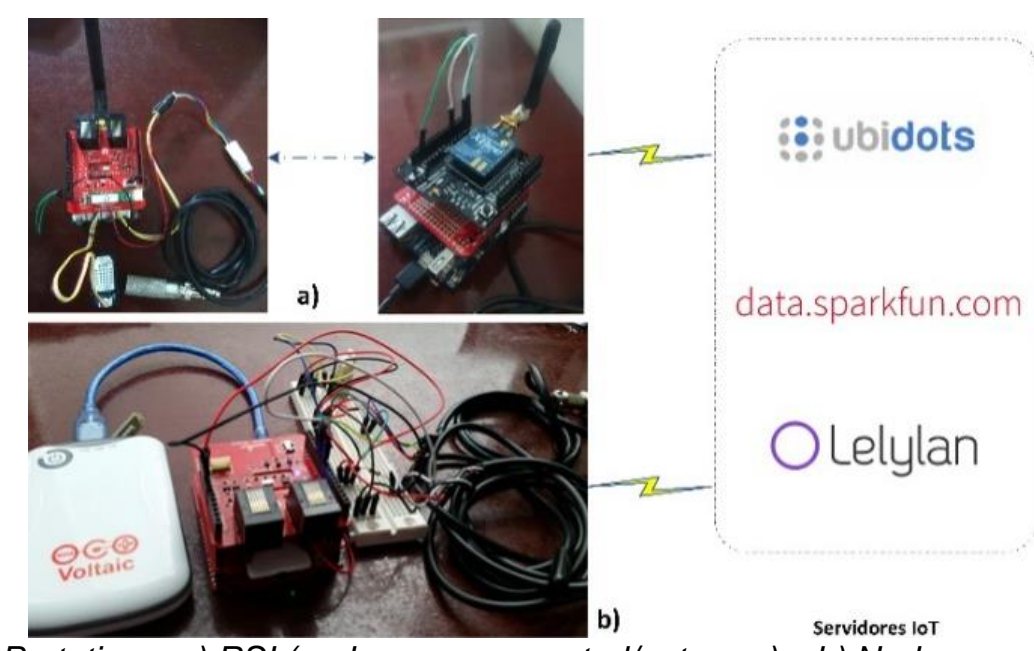

Figura 4. Prototipos: a) RSI (nodo sensor y central/gateway) y b) Nodo sensor usando Wi-Fi. 
Tabla 2. Requerimiento diario de potencia de los nodos.

\begin{tabular}{ll|rrrr}
\hline Nodo & Proceso & Consumo (mA) & Tiempo (s) & Potencia (mWh) & Pot. diaria (Wh/día) \\
\hline \multirow{3}{*}{ Sensor } & Adquisición & 60 & 30 & 204.30 & 4,90 \\
\cline { 2 - 6 } & Transmisión & 70 & 2 & 16,17 & 0,388 \\
\cline { 2 - 6 } & Total & & & & $\mathbf{5 , 2 8}$ \\
\hline \multirow{3}{*}{ Central } & Activo & 49 & 15 & 90,41 & 2,17 \\
\cline { 2 - 6 } & Recepción & 130 & 10 & 170,3 & $\mathbf{4}, 08$ \\
\cline { 2 - 6 } & Transmisión & 165 & 2 & 43,96 & $\mathbf{7 , 3}$ \\
\cline { 2 - 6 } & Total & & & 91,97 & 2,207 \\
\hline \multirow{2}{*}{ Sensor } & Adquisición & 25 & 600 & 0,675 & 0,088 \\
Wi-Fi & Transmisión & 109 & 6 & & $\mathbf{2 , 2 9 5}$ \\
\cline { 2 - 6 } & Total & & & & \\
\hline
\end{tabular}

El consumo diario de energía se calcula mediante la ecuación (1), considerando la carga conectada y la eficiencia del regulador (regulador interno del Arduino Uno) en este caso del 95\% (Quezada, Morales \& Riofrío, 2013).

$$
E_{d}=\frac{P_{d}}{\eta_{\text {reg }}}
$$

Dónde: $\mathrm{E}_{\mathrm{d}}$, es el consumo diario de energía del prototipo de adquisición y transmisión en Wh/día, $P_{d}$ es el requerimiento diario de potencia de los dispositivos a alimentar en Wh/día, $\eta_{\text {reg }}$ es la eficiencia del regulador en \%. En la Tabla 3 se muestra el $E_{d}$, de cada uno de los nodos.

Tabla 3. Requerimiento diario de potencia de los nodos.

\begin{tabular}{rrrr}
\hline & Nodo Sensor & Nodo central & Nodo Sensor Wi-Fi \\
\hline Ed(Wh/día) & 5,55 & 7,7 & 2.415 \\
\hline
\end{tabular}

La medición del consumo energético de las pruebas experimentales se realiza en intervalos de 30s (ver Figura 5); el intervalo de tiempo de transmisión recomendado por la OMM está entre 5 a 10 min (OMM, 2014). Con estos intervalos de tiempo en la transmisión el consumo de energía es menor, permitiendo incrementar la autonomía del prototipo.

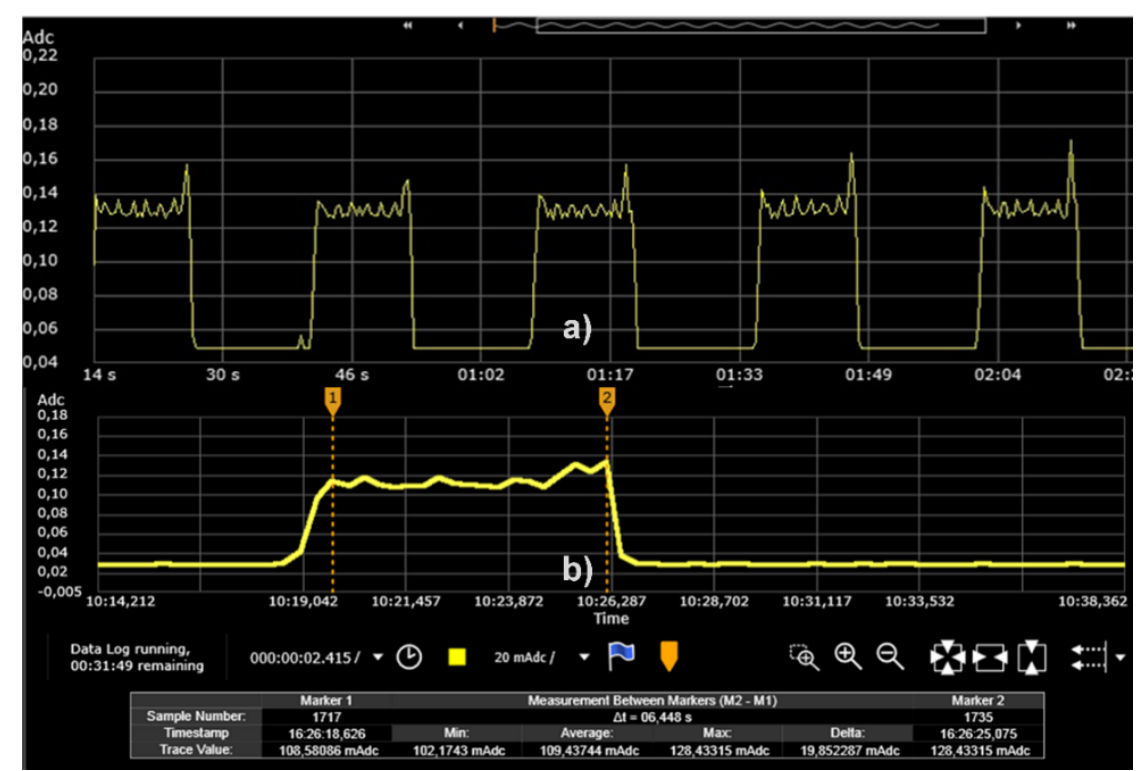

Figura 5. Consumo de corriente: a) nodo central y b) nodo sensor Wi-Fi. 


\subsubsection{Dimensionamiento del panel solar}

Para dimensionar correctamente el número de paneles solares y la capacidad necesaria de los mismos, se toma como referencia los parámetros de consumo diario de potencia de los prototipos (Wh/día) y la irradiación global diaria del lugar $(\mathrm{kWh} / \mathrm{m} 2 / \mathrm{d})$. Se utiliza una metodología para el dimensionado de sistemas fotovoltaicos autónomos, para determinar el panel solar y batería (Aguilera \& Hontario, 2011; Prat, 2015). Para poder obtener la irradiación global del lugar de interés se utilizó recursos web del Data Center de la NASA en el área de Meteorología y Energía Solar (Stackhouse, 2016), donde se ingresa las coordenadas de latitud y longitud de la ciudad de Loja-Ecuador. En la Tabla 4, se muestra todos los parámetros utilizados para el dimensionamiento del panel solar.

Tabla 4. Parámetros para el cálculo del panel solar.

\begin{tabular}{|c|c|c|}
\hline Parámetros & Unidad & Valor \\
\hline Constantes Solar ( $\left.\mathrm{L}_{\mathrm{cs}}\right)$ & $\mathrm{W} / \mathrm{m}^{2}$ & 1367 \\
\hline Día central del mes $\left(d_{n}\right)$ & $\mathrm{d}$ & 15 \\
\hline Corrección Excentricidad orbita $\left(\mathrm{E}_{0}\right)$ & & 1.319 \\
\hline Latitud (grados decimales) & $\left({ }^{\circ}\right)$ & -3.98 \\
\hline Longitud ( $\varphi$ : grados decimales) & $\left({ }^{0}\right)$ & -79.16 \\
\hline Angulo diario $(\Gamma)$ & Rad & 0.2409 \\
\hline Declinación Solar $(\delta)$ & & -0.3712 \\
\hline Angulo de salida del sol $\left(\omega_{s}\right)$ & Rad & 1.5978 \\
\hline $\begin{array}{l}\text { Irradiación solar extra terrestre sobre superficie horizontal } \\
\left(G_{0 d}\right)\end{array}$ & $\mathrm{kWh} / \mathrm{m}^{2} / \mathrm{d}$ & 13.3551 \\
\hline $\begin{array}{l}\text { Irradiación global diaria promedio mensual en una } \\
\text { superficie horizontal }\left(G_{d}\right)\end{array}$ & $\mathrm{kWh} / \mathrm{m}^{2} / \mathrm{d}$ & 5.07 \\
\hline Índice de transparencia atmosférica $\left(\mathrm{K}_{\mathrm{d}}\right)$ & & 0.3796 \\
\hline Irradiación difusa en la superficie horizontal ( $\left.D_{\mathrm{d}}\right)$ & $\mathrm{kWh} / \mathrm{m}^{2} / \mathrm{d}$ & 2.475 \\
\hline Irradiación directa en superficie horizontal $\left(\mathrm{I}_{\mathrm{d}}\right)$ & $\mathrm{kWh} / \mathrm{m}^{2} / \mathrm{d}$ & 2.5942 \\
\hline
\end{tabular}

Con los resultados obtenidos de la capacidad de almacenamiento de la batería mínima requerida (ver tabla 5), se determina el uso de baterías de tipo LiPo de 6600 mAh a 3.7V. Además, en base al dimensionamiento del panel solar se utiliza uno de $5.2 \mathrm{~W}$ (el mínimo requerido es de $3.2 \mathrm{~W}$ ).

Tabla 5. Resultados del dimensionamiento del panel solar, inclinación y HPS.

\begin{tabular}{lccc}
\hline Parámetros & RSI DigiMesh & \multicolumn{2}{c}{ RSI Wi-Fi } \\
\cline { 2 - 4 } & Nodo central & Nodo Final & Nodo Sensor \\
\hline Número de Paneles $\left(\mathrm{N}_{\mathrm{T}}\right)$ & 0.7672 & 0.553 & 0.240 \\
\hline Potencia $(\mathrm{W})$ & 3.2 & 3.2 & 3.2 \\
\hline Ángulo Inclinación $\left(^{\circ}\right)$ & 30 & 30 & 30 \\
\hline Hora pico solar $(\mathrm{HPS})$ & 3.92 & 3.92 & 3.92 \\
\hline
\end{tabular}

Para la implementación del sistema de abastecimiento de energía, se necesita cargar la batería con el panel solar utilizando un regulador/cargador solar MPPT (Maximum Power Point TrackingSeguidor de Punto de Máxima Potencia) para baterías litio (ver Figura 6). Éste componente permite obtener eficientemente la energía del panel solar. 


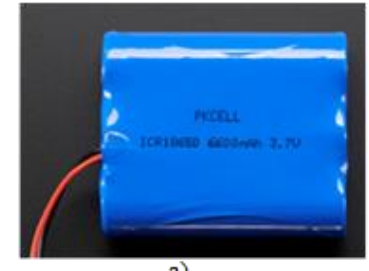

a)

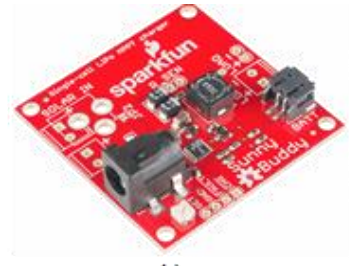

b)

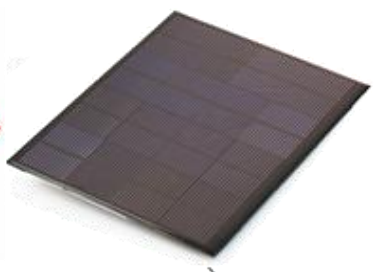

c)

Figura 6. Componentes del sistema de abastecimiento de energía solar: a) Batería LiPo, b) cargador solar y

c) panel Solar.

\section{Resultados y Discusión}

\subsection{Despliegue de prototipo}

Para preservar el correcto desempeño de los componentes del prototipo recolector de datos meteorológicos y obtener las mediciones exactas, se adecuan los componentes en una caja con un grado de protección IP65 o superior (Kuppusamy, 2016).

Tomando en cuenta las recomendaciones que pone a consideración la OMM (OMM, 2014), el prototipo se instala en un campo abierto dentro del campus universitario de la UTPL (ver figura 7). Para la obtención de las variables meteorológicas en tiempo real se usa la RSI basado en DigiMesh. La distancia entre el nodo central y el nodo sensor es de 190m.
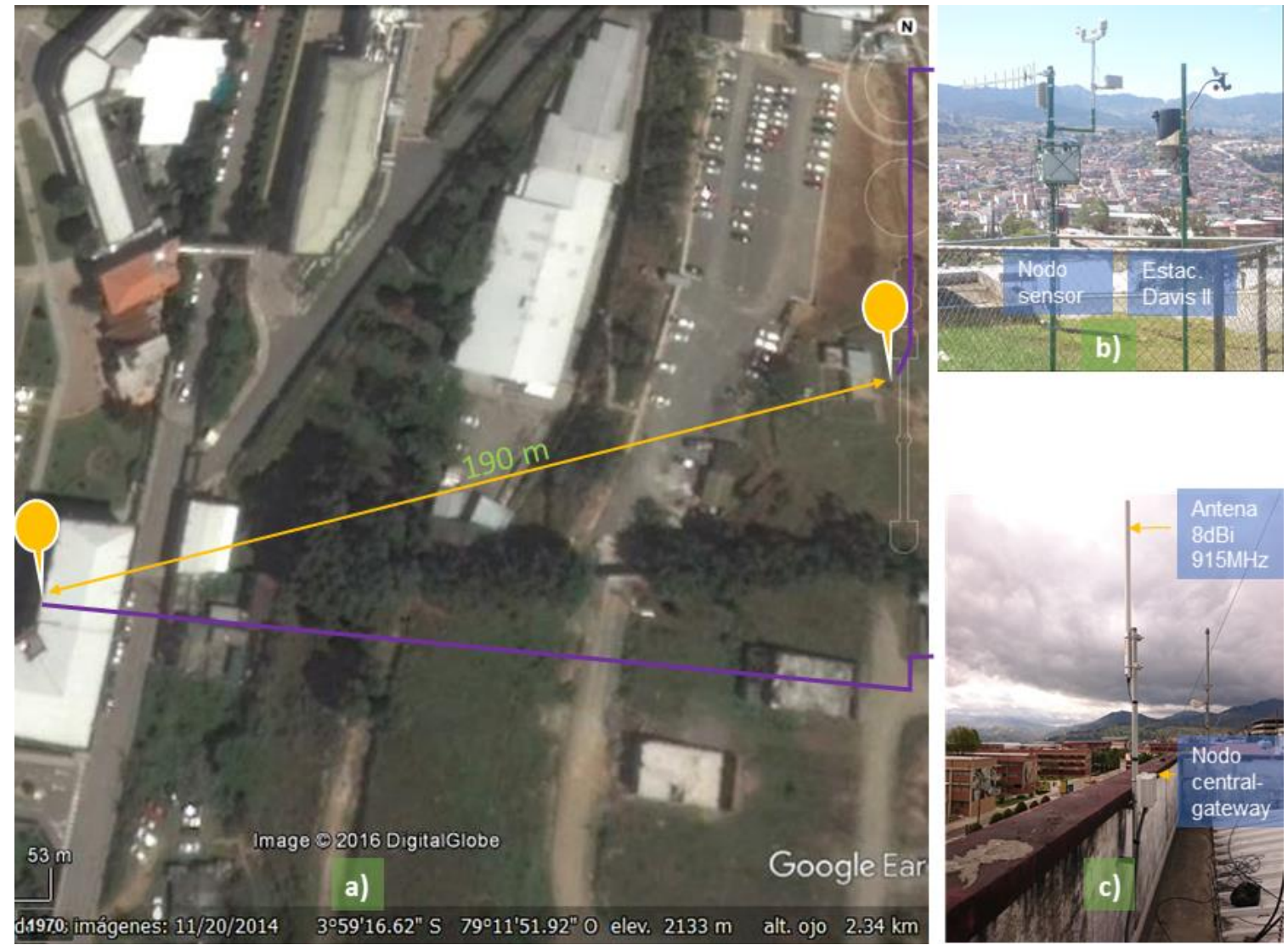

Figura 7. Despliegue de la RSI: a) Ubicación nodo sensor y central, b) Nodo sensor y c) Nodo central. 


\subsection{Pruebas de cobertura del sistema}

Se realiza una prueba cobertura de la RSI DigiMesh con el propósito de incrementar a futuro la cantidad de nodos sensores. Para este propósito se ha empleado el nodo central de la Figura 7.c y se ha implementado un nodo sensor portátil (ver Figura 8a).

Para la prueba se ha establecido un enlace con una distancia de $7 \mathrm{Km}$ (ver figura $8 \mathrm{~d}$ ) entre el nodo central y un nodo sensor portátil ubicado en la Central Eólica Villonaco (CEV) CELEC EP GENSUR (lugar con línea de vista hacia UTPL ver figura 8b) hasta el nodo central ubicado en la UTPL como se observa en la figura 8c. El nodo sensor portátil cuenta con una antena direccional tipo Yagi de $14 \mathrm{dBi}$, el nodo central con una omnidireccional de $8 \mathrm{dBi}$ y se ha empleado una potencia de transmisión de $17 \mathrm{dBm}$ o $50 \mathrm{~mW}$ en cada módulo de comunicación de los nodos.

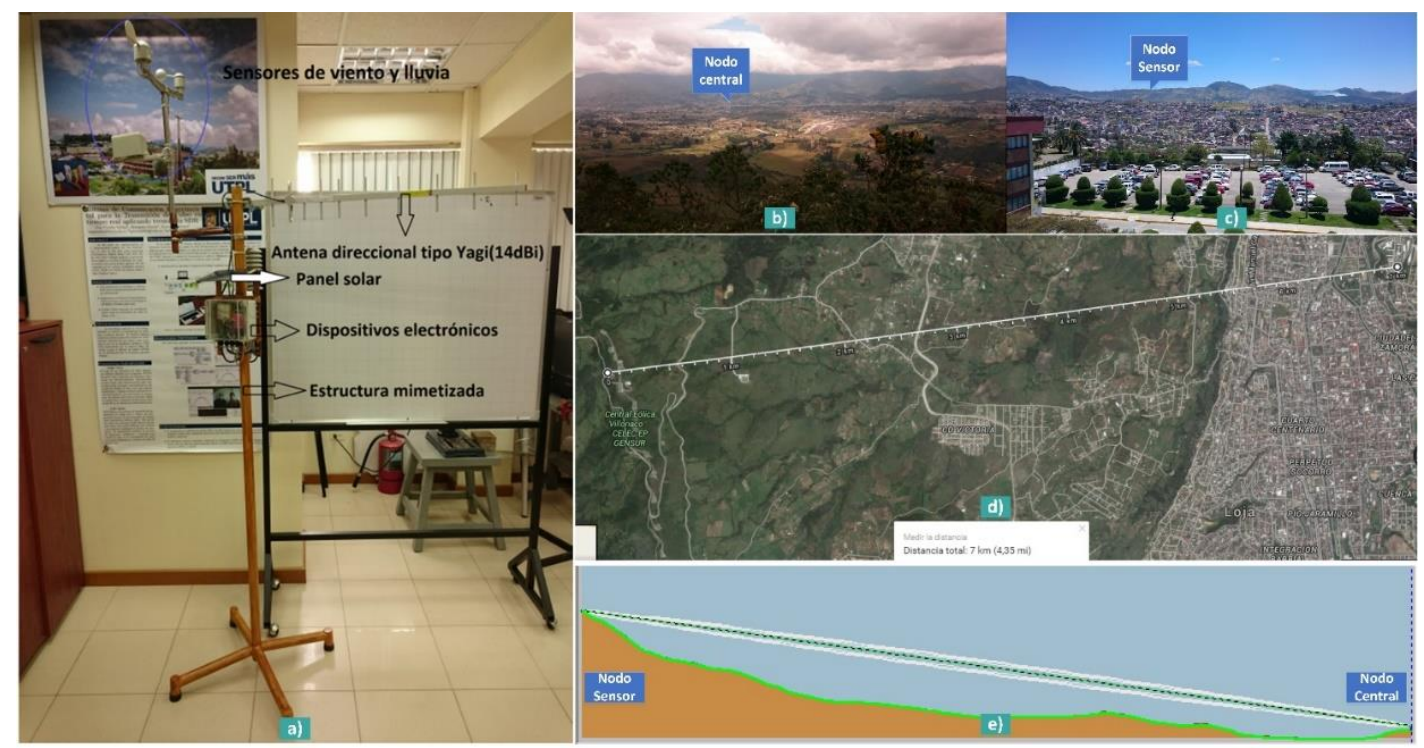

Figura 8. Prueba de cobertura del sistema a una distancia de 7km: a) Nodo sensor portátil, b) Línea de vista CEV - UTPL, c) Línea de vista UTPL - CEV, d) Distancia entre los nodos, y e) Perfil del enlace.

Para verificar que existe línea de vista y que la primera zona de Fresnel está despejada se usó el software de simulación RadioMobile, el resultado obtenido se observa en la figura 8e, estableciéndose el enlace. Con esta prueba de cobertura se determina que se puede extender la RSI DigiMesh, para la instalación de nodos con sensores medioambientales para obtener datos en tiempo real de manera continua y a largo plazo de áreas de interés como la Reserva de Biosfera Podocarpus que es fuente de 4 ríos binacionales que abastecen de agua a casi 2 millones de personas en Ecuador y Perú (NATURALEZAYCULTURA, 2016); y de bosques protectores altos de montaña que se encuentran a las afueras de la ciudad de Loja que son la principal fuente de agua para la ciudad.

\subsection{Visualización de datos en plataformas loT}

Para el almacenamiento de los datos en las plataformas de loT, se requiere de la construcción de una trama JSON que demanda cada plataforma. Un ejemplo de esto es la plataforma 
DataSparkfun donde se necesita usar una llave privada y una llave pública. En la Figura 9 se muestra la publicación de los datos recolectados por el prototipo desplegado en los servidores de Ubidots, ThingSpeak y DataSparkfun (Phant).

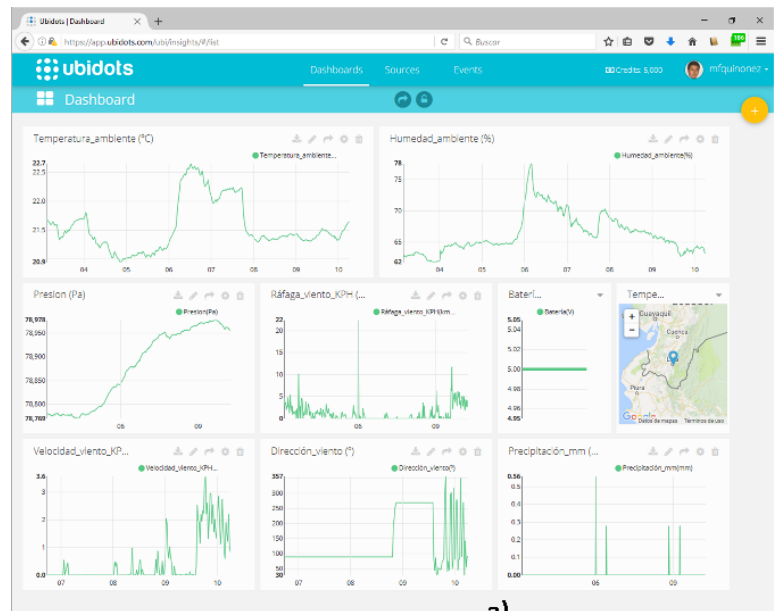

a)
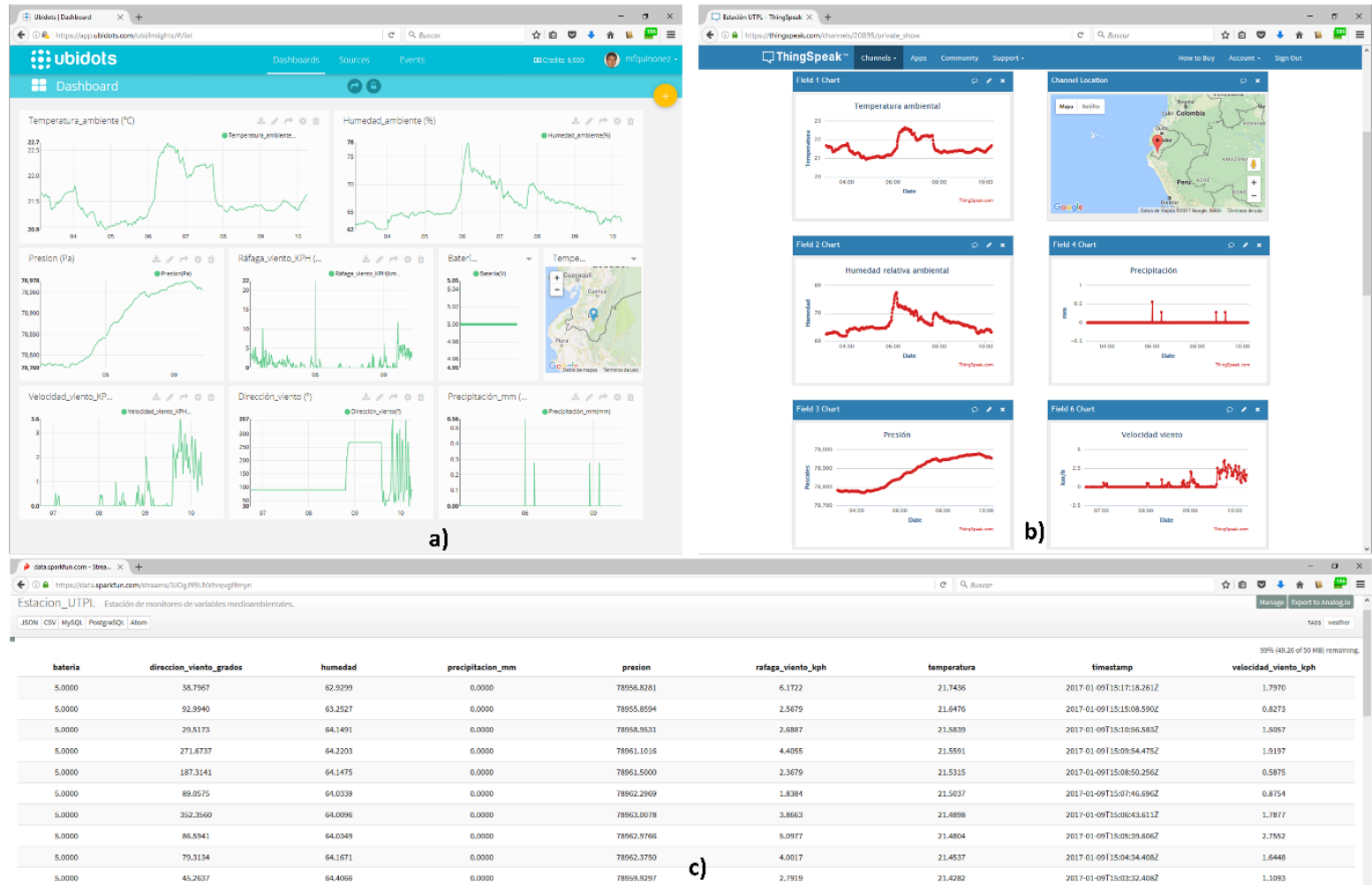

Figura 9. Publicación de datos en las plataformas de loT: a) Ubidots, b) ThingSpeak y c) DataSparkfun.

Estas plataformas de loT, permiten el almacenamiento masivo de datos, la visualización y análisis, siendo accesible a usuarios con diferentes propósitos como estudios climatológicos o toma de decisiones. A pesar de las ventajas mencionadas, estas tres plataformas presentan limitantes con respecto a la cantidad de datos que se puede almacenar, número de dispositivos conectados y la retención de datos. Por lo que es necesario analizar si es conveniente el pago de estos servicios dependiendo de la cantidad de nodos y variables o si es necesario desarrollar una plataforma que integre MQTT y que permita la interoperabilidad usando estándares como SWE (Sensor Web Enablement) de OGC (Open Geospatial Consortium) (OGC, 2016).

\subsection{Análisis de datos}

Para la obtención de las variables meteorológicas en tiempo real se usa la RSI basado en DigiMesh. En la Figura 10 se muestran los datos obtenidos de los sensores de temperatura ambiente y humedad relativa ambiente de tres días desde el 7 al 9 de enero del 2017.

Para validar los resultados obtenidos del prototipo se realiza una comparación y análisis de los datos con una estación meteorológica Davis Vantage Pro (ver figura 7b). El análisis corresponde a determinar el error relativo entre los grupos de datos. Estos datos corresponden a un conjunto de 490 muestras realizadas el 7 de enero desde las 18:00 a 8:40 del 9 de enero de 2017. 


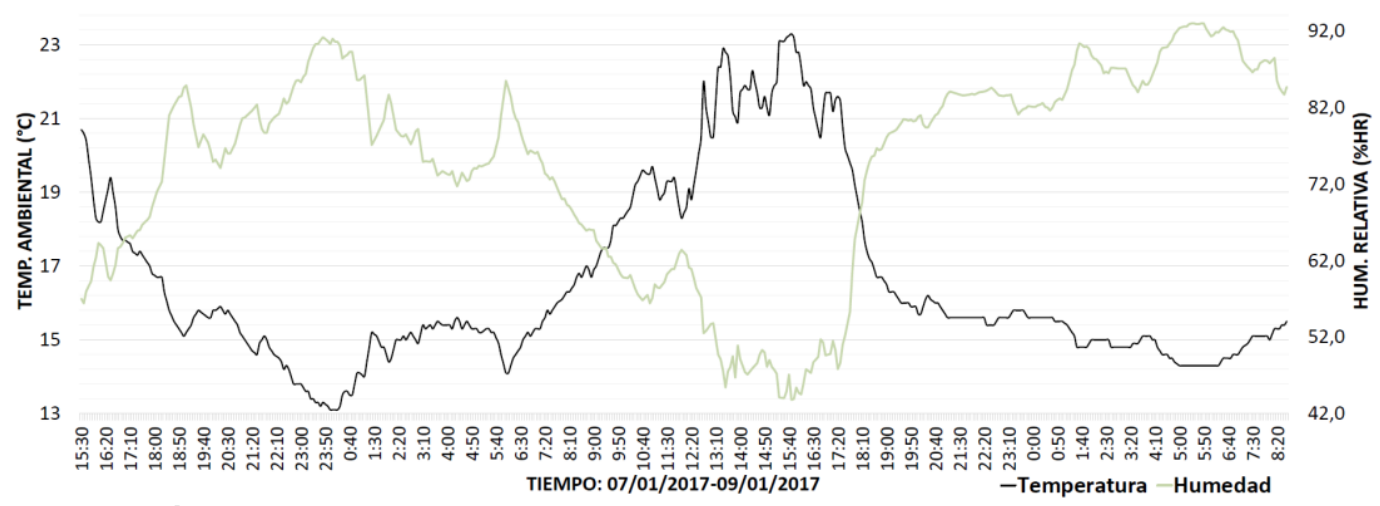

Figura 10. Datos de temperatura y humedad obtenidos por el sistema.

En la Figura 11a se muestra la gráfica de los datos correspondientes a la temperatura ambiental y el error relativo. Obteniendo un error máximo de 5,7\%. En la Figura $11 b$ se muestra la humedad ambiental y el error relativo. El error máximo es de 5,17\%.

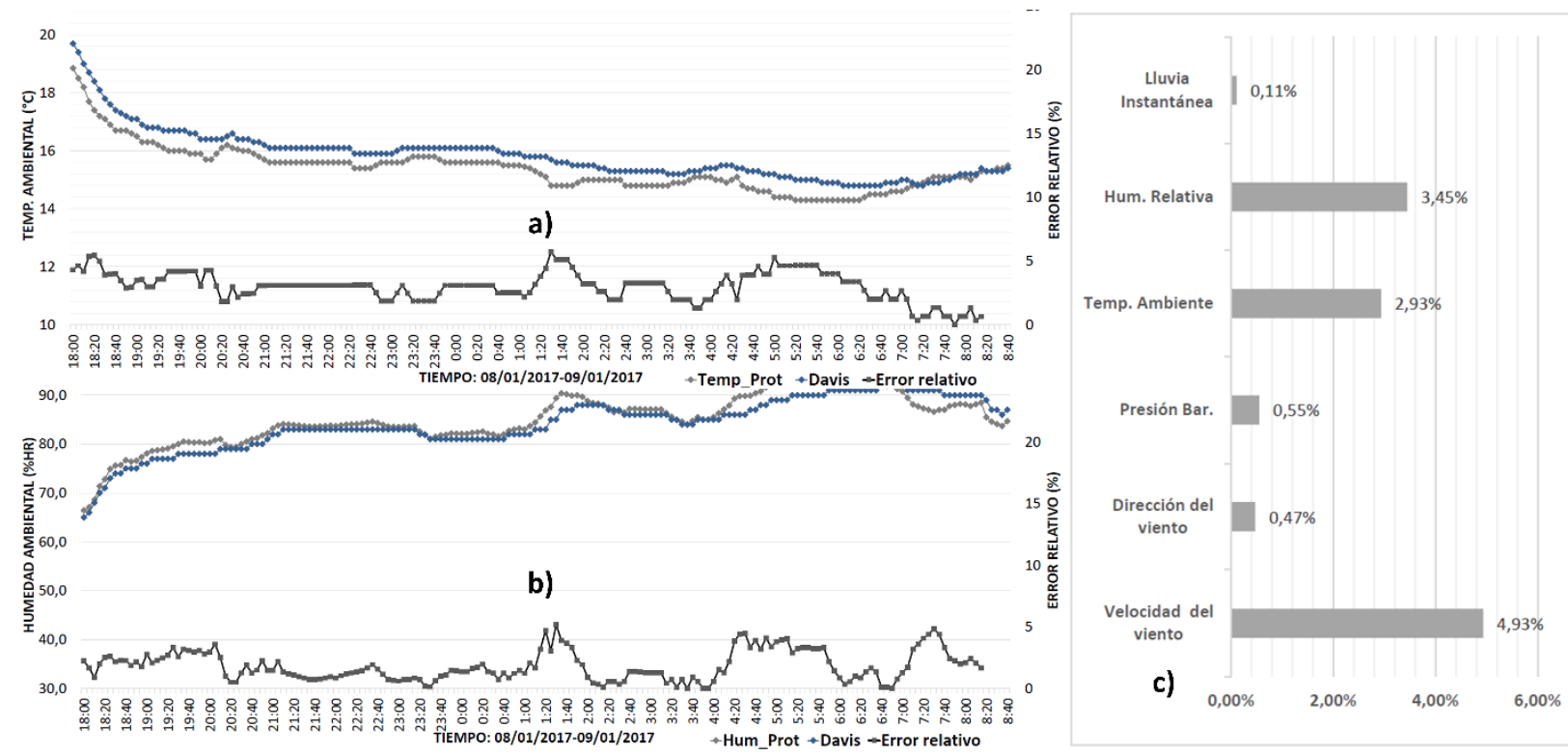

Figura 11. Análisis de datos: a) Temperatura ambiental y error relativo, b) Humedad ambiental y error relativo y c) Error relativo promedio de las variables medioambientales.

Para las variables restantes los errores relativos promedios se hallan entre $0.11 \%$ y $4.93 \%$ (ver Figura 11c).

\section{Conclusiones y Recomendaciones}

Se ha desarrollado un sistema de monitoreo que automatiza el proceso de obtención de datos en tiempo real de variables medio ambientales recomendadas por la OMM usando una RSI y plataformas de internet de las cosas, que facilitan el acceso a la información y su gestión, usando plataformas de hardware y software abiertas, de bajo costo que permiten la integración o cambio de módulos de sensores o de comunicación, para incrementar las funcionalidades del prototipo, empleando protocolos de comunicación estándar que facilitan la escalabilidad y reconfiguración de los nodos. Además, cuenta con un sistema de abastecimiento de energía solar y de 
almacenamiento, que garantiza de manera continua y a largo plazo su funcionamiento; e incluye la transmisión de datos en tiempo real usando tecnologías inalámbricas.

Al usar DigiMesh en el sistema se logró una cobertura de $7 \mathrm{~km}$, logrando cubrir varios bosques altos de montaña como la Reserva de Biosfera Podocarpus que es una de las principales fuentes de recurso hídrico de la ciudad de Loja. Además, el sistema permite flexibilidad para expandir la red, con la capacidad de integrar más nodos y crear una red tipo malla.

\section{Bibliografía}

Asghar, M. \& Mohammadzadeh, N. (2015). Design and simulation of energy efficiency in node based on MQTT protocol in Internet of Things. 2015 International Conference On Green Computing And Internet Of Things (ICGCIOT). http://dx.doi.org/10.1109/icgciot.2015.7380689

Aguilera, J. \& Hontario, L. (2011). Dimensionado de sistemas fotovoltaicos autónomos [en línea]. Departamento de Electrónica, Escuela Politécnica Superior, Universidad de Jaén. Jaén (España): Grupo IDEA <https://manuelberaun.files.wordpress.com/2011/12/dimensionadode-sfv-autonomos.pdf> [consulta: 1/4/16].

Bolivar, I. \& Alexandre da Silva, G. (2015). Solar radiation monitoring using electronic embedded system Raspberry Pi database connection MySQL, Ubidots and TCS-230 sensor. 2015 CHILEAN Conference On Electrical, Electronics Engineering, Information And Communication Technologies (CHILECON). http://dx.doi.org/10.1109/chilecon.2015.7400420

Faludi, R. (2011). Building wireless sensor networks (1st ed.). Beijing: O'Reilly. 392 p. ISBN: 9780-596-80773-3.

Kuppusamy, P. (2016). Smart Home Automation using Sensors and Internet of Things. Asian Journal Of Research In Social Sciences And Humanities, 6(8), 2642. http://dx.doi.org/10.5958/2249-7315.2016.00771.1

Lin, C., Zadorozhny, V., Krishnamurthy, P., Park, H., \& Lee, C. (2011). A Distributed and Scalable Time Slot Allocation Protocol for Wireless Sensor Networks. IEEE Transactions On Mobile Computing, 10(4), 505-518. http://dx.doi.org/10.1109/tmc.2010.163

MATHWORKS (2016). Understand Your Things, the open IoT platform with MATLAB analytics. [en línea]. ThingSpeak: < https://thingspeak.com/ > [consulta: 7/4/16]. 
NATURALEZAYCULTURA (2016). Reserva de Biosfera Podocarpus - El Cóndor [en línea]. Loja (Loja, $\quad$ Ecuador): Naturaleza y Cultura Internacional <http://www.naturalezaycultura.org/spanish/htm/ecuador/areas-andes-podocarpus.htm> [consulta: $2 / 11 / 16]$.

OMM (2014). Guía de instrumentos y métodos de observación meteorológicos [en línea]. Ginebra (Suiza): OMM <http://l ibrary.wmo.int/pmb_ged/wmo_8-2014_es.pdf> [consulta: 10/09/16].

Perera, C., Liu, C., \& Jayawardena, S. (2015). The Emerging Internet of Things Marketplace From an Industrial Perspective: A Survey. IEEE Transactions On Emerging Topics In Computing, 3(4), 585-598. http://dx.doi.org/10.1109/tetc.2015.2390034

PHANT (2015). Documentation, phant.io. Colorado (Boulder, CO, United States): SparkFun Electronics <http://phant.io/docs/> [consulta: 5/4/16].

Prat, I. (2015). Dimensionado de Sistemas Fotovoltaicos [en línea]. Universitat Politécnica de Catalunya. Barcelona (España): Departamento de Ingeniería Electrónica <http://datateca.unad.edu.co/contenidos/358054/dimensionado_sistema_fotovoltaico.pdf> [consulta: 3/4/16].

OGC (2009). Sensor Web Enablement (SWE) [en línea]. Massachusetts (Wayland, USA) http://www.opengeospatial.org/ogc/markets-technologies/swe [consulta: 2/12/16].

Quezada, V., Morales, I. E., \& Riofrío, I. (2013). Diseño y fabricación de un equipo portable para provisión de energía eléctrica basado en el aprovechamiento de energía solar, orientado a aplicaciones de camping [en línea]. Repositorio Institucional de Trabajos de fin de Titulación de la Universidad Técnica Particular de Loja. Loja (Loja, Ecuador): UTPL < http://dspace.utpl.edu.ec/handle/123456789/7865> [18/07/2015].

Stackhouse, P. (2016). NASA Surface meteorology and Solar Energy: RETScreen Data. USA: Atmospheric Science Data Center <https://eosweb.larc.nasa.gov/cgibin/sse/retscreen.cgi?email=rets\%40nrcan.gc.ca\&step=1\&lat=-3.987667\&lon=79.196763\&submit=Submit.> [consulta: 5/07/16].

UBIDOTS (2014). Ubidots API Documentation [en línea]. Bogotá (Colombia): UBIDOTS $<$ http://ubidots.com/docs/get_started/overview.html> [consulta: 3/4/16].

Wong, B. \& Kerkez, B. (2016). Real-time environmental sensor data: An application to water quality using web services. Environmental Modelling \& Software, 84, 505-517. http://dx.doi.org/10.1016/j.envsoft.2016.07.020 